\title{
Geometrical Elaboration of Auxetic Structures
}

\author{
Milena Stavric $^{1}$ (D) Albert Wiltsche $^{1}$
}

Published online: 18 January 2019

(c) The Author(s) 2019

\begin{abstract}
This paper analyzes the basic geometric and kinematic characteristics of auxetic structures. The geometric principles are then transferred to a family of new possible forms. We investigate and elaborate auxetic behavior in a purely geometric way which is based on the kinematic movement of different frameworks. We then demonstrate its usefulness by analyzing the involved geometry with computer software but without computer simulations or numerical approximations. Instead, using cut flat material and, depending on the cuts and the material used, we enable the kinematical movement of the structures. We also analyze monostable auxetic structures whose movements can be described as geometrically precise, as well as bistable ones. Based on geometric considerations, we combine rigid materials and composites and select appropriate joint connections to allow the application of this system in an architectural scale in, for example, facades, screens or shading systems.
\end{abstract}

Keywords Auxetics $\cdot$ Kinematics $\cdot$ Fabrication

\section{Introduction}

When elastic material is stretched, it becomes longer in the direction of stretching and is typically thinner in the orthogonal direction. The behavior of material under such a deformation is described by one of the fundamental mechanical termsPoisson's ratio (Evans et al. 2000). Poisson's ratio $(\mu)$ defines how a material expands (or contracts) transversely when being compressed longitudinally. Most natural materials have a positive $\mu$ indicative of our intuitive understanding that material becomes thinner when it is stretched. Auxetic materials however, can

Milena Stavric

mstavric@tugraz.at

Albert Wiltsche

wiltsche@tugraz.at

1 Institute of Architecture and Media, Graz University of Technology, Kronesgasse 5, 8010 Graz, Austria 
exhibit a negative Poisson's ratio. Such materials undergo lateral expansion when stretched longitudinally and become thinner when compressed (Ellul et al. 2009).

The behavior of auxetic materials is of great interest for material sciences and most auxetic research is done on microscale and mesoscale. The application of such materials includes medical implants of expandable stents (Gatt et al. 2015; Najabat et al. 2014; Tan et al. 2011) through to applications in sports fabrics (Sanami et al. 2014) to programmable materials that can form different shapes autonomously by folding (Hawkes et al. 2010). Such material concepts are experimentally realized by perforating various cut motifs into a sheet of material creating a network of solids and voids. Thin parts of material that connect solids act as hinges and allow auxetic behavior of the whole system. Furthermore, we can distinguish between monostable and bistable auxetic mechanical materials. The main mechanical properties of a monostable material is that such auxetics cannot maintain the transformed shape upon load removal whereas bistable auxetic materials have two stable positionsexhibiting a switchable expandability (Rafsanjani and Pasisi 2016).

The existing research in the material sciences is strongly based on experiments, computer simulations and numerical approximations of auxetic capabilities. Only a small number of research studies give a precise geometric description of auxetic structures (Borcea and Streinu 2015; Elipe and Lantada 2012) or describe possible applications for design purposes (Konakovic et al. 2016; Mesa 2016). For example, the research of Borcea and Streinu (2015) presents purely geometric notions of auxetic one-parameter deformations of periodic 2D and 3D frameworks. But from a design point of view, this gives no framework for further development or a hint of how to find new auxetic designs with one-parameter deformations. Elipe and Lantada (2012) records the results of a comparative study of planar auxetic geometries by means of computer-aided design and engineering. They develop a library of existing different auxetic structures and simulate the behavior of different auxetic geometries and elaborate properties of the auxetic structures.

Our work will go further because we will analyze the geometry of auxetic structures in order to define classes of possible auxetic patterns. Based on the seventeen wall paper groups-especially on Platonic and Archimedean patterns and the associated Euclidian transformations-we will analyze periodical patterns and develop additional a-periodical patterns in order to construct a network with a continuous change of the auxetic volume. Our parametric approach will enable generation of such patterns performed in an appropriate CAD system. With a series of parametric design studies and physical prototypes we will demonstrate that our approach encompasses a rich class of periodical and a-periodical patterns that, apart from potential use in material science, can be applied as kinematic systems in engineering and design for performative architecture.

\section{Auxetics}

Figure 1 shows the typical behavior of elastic material. When forces $F_{1}$ and $F_{2}$ are applied in two opposite (longitudinal) directions on the material, it is compressed in the transverse direction. The new volume $\mathrm{V}_{1}$ usually becomes smaller or at most 


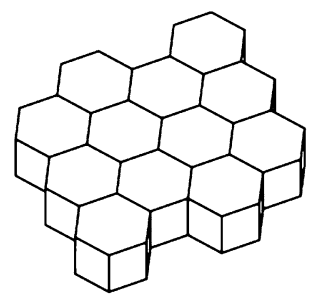

V

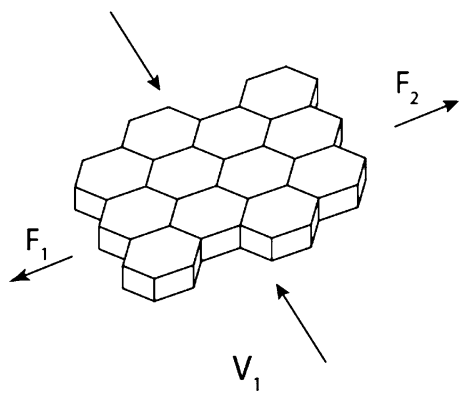

Fig. 1 Typical elastic material with volume $\mathrm{V}$ has positive Poisson's ratio which means that it contracts lateral to the direction of applied forces $F_{1}$ and $F_{2}$. The contracted volume $V_{1}$ usually gets smaller, so $\mathrm{V}_{1} \leq \mathrm{V}$

the same as $\mathrm{V}$ which means $\mathrm{V}_{1} \leq \mathrm{V}$. The ratio between the negative transverse strain divided by the longitudinal one is called Poisson's ratio. Since typical materials contract in transverse direction, when stretched in longitudinal one, Poisson's ratio is positive and approximately between 0 and 0.5 (Lakes 1987).

Lakes (1987) was the first to develop research about auxetics in science. Auxetics are forms whose geometric structure is not completely rigid. The deformation means a change of geometry, whereby the structure changes from a relative stable state into a moveable kinematic state. Auxetic materials show a completely opposite behavior compared to general elastic material, since it is also stretched in the transverse direction of the applied forces and so the volume increases and Poisson's ratio becomes negative, due to its definition. But in reality, the material's volume is not really increasing. Namely, the whole structure is composed of different smaller parts which rotate against each other when forces are applied. So "only" the overall volume-which means the volume of the bounding box-becomes larger (Fig. 2). Hence, the whole system is considered as a combination of materialized solid parts and the space (gap or
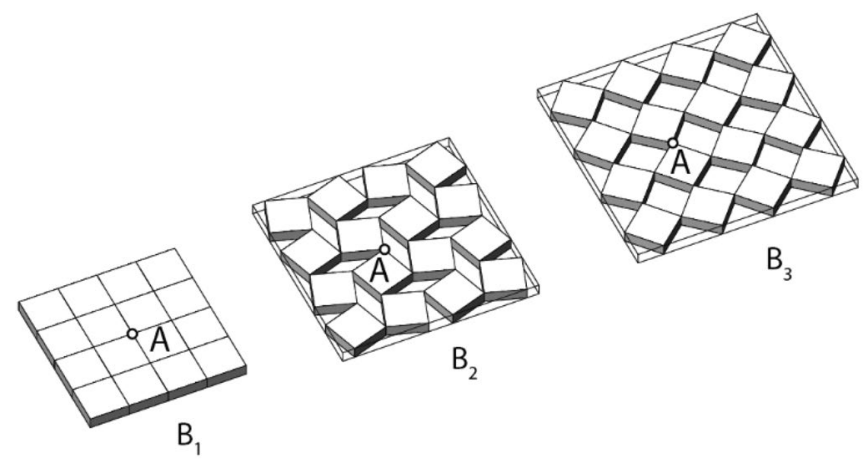

Fig. 2 An auxetic structure which is composed by smaller solid parts increases its overall volume by rotating the parts against each other. This leads to bigger bounding boxes $B_{1}<B_{2}<B_{3}$ 

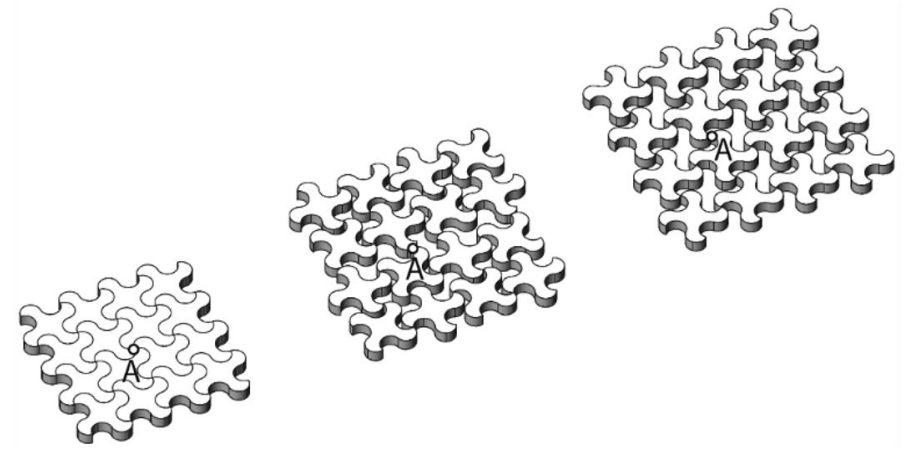

Fig. 3 A variation of the simple pattern in Fig. 2 yields the pattern p4

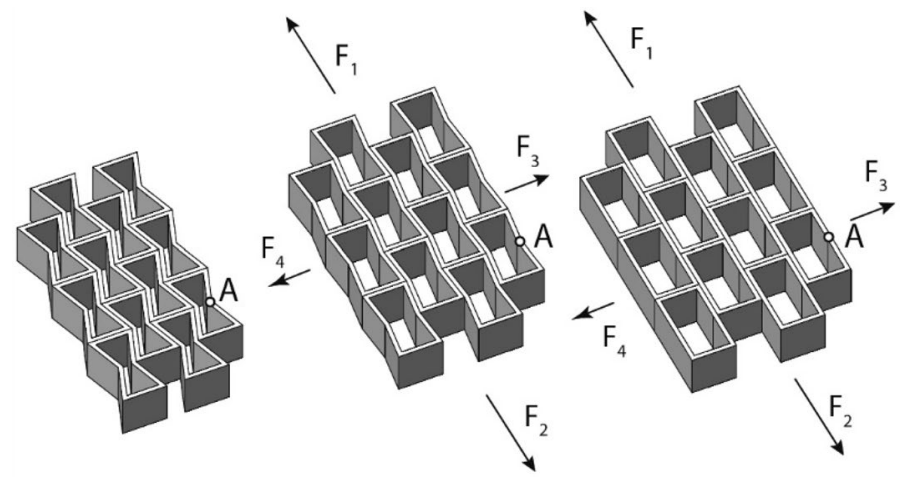

Fig. 4 A famous auxetic structure. Created when applying the shown forces, the structure increases its overall volume

void) in between. A detailed discussion of the basic geometrical principles of such rigid structures is contained in the work of Mesa et al. (2017). In current paper one also can find architectural design made of elastic polyurethane foam.

From a designer's point of view it is very important to have variations of geometric forms, since design tasks are always different and the nature of design is to be unique. So our approach is to take simple geometric patterns-like the one in Fig. 2-and check it for auxetic behavior. In a second step we vary the design of the pattern based on the input geometry. One result of this can be seen in Fig. 3.

A well known auxetic structure is shown in Fig. 4, since it is one of the oldest investigated forms. From a geometric point of view, it consists "only" of boxshaped solid side parts that are connected by revolute joints. All the axes of the joints in the figure are parallel and vertical. It deforms from a smaller form consisting of "butterfly" shaped hexagon prisms on the left side to rectangular boxes. 


\section{Monostable and Bistable Auxetics}

From our geometric point of view it is important to distinguish between two auxetic forms, since we want to describe the motion of the structure in a precise kinematic way (Bottema and Roth 1979).

\section{Monostable Auxetics}

A "monostable auxetic" is a moveable structure, which allows a continuous, planar (or spatial), one degree of freedom motion (Bottema and Roth 1979). It changes its overall size (or volume) when it is moved. Since we concentrate on the three regular Platonic and eight semi-regular Archimedean patterns and generally on the seventeen wallpaper-groups (Pottmann et al. 2007) we investigate the ones which show monostable auxetic behavior. Some of the groups can be used to design oneparametrical planar one degree of freedom motions. There are also ornamental patterns which allow us to implement parallel or even diamond-shaped four-bar linkages in between the pattern parts. The pattern in Fig. 2 can be used to implement parallel four-bar linkages (diamond-shaped). Figure 5 shows different positions of the pattern and one of its parallel four-bar linkages. A four-bar linkage describes a planar kinematic motion with a one parameter degree of freedom (Bottema and Roth 1979). The shown system is a combination of four-bar linkages that are interdependently linked. Due to the symmetric configuration the systems stays movable and keeps its one degree of freedom.

One of the drawbacks of the system in Fig. 5 is that the hinges collide in the closed position. Therefore, a practical implementation is restricted to open positions of the systems or it must be realized by means of special hinges (see later section on hinges, and Fig. 14) or a design shown in Fig. 7.

Another version of this system is demonstrated in Figs. 6 and 7. In this case we use a parallel four-bar linkage instead of a diamond-shaped one. This allows us to construct a system where we start from a closed version of the pattern and end in a closed one and the hinges do not collide.

In order to enable the system to close, we have to ensure that the short edge of the parallelogram is half of the length of the longer one. For practical purposes, we have to cut gaps into the pattern parts in order to create an empty place for

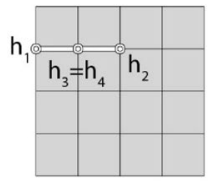

(a)

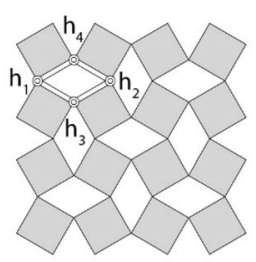

(b)

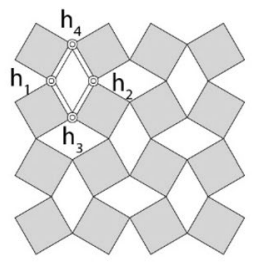

(c)

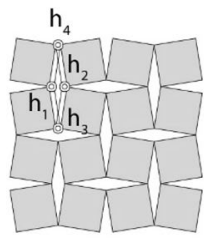

(d)

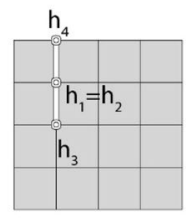

(e)

Fig. 5 Planar moveable ornamental pattern with one marked parallel four-bar linkage and its revolute hinges $h_{i}$ 


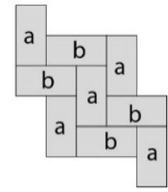

(a)

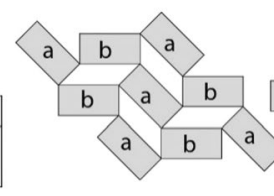

(b)

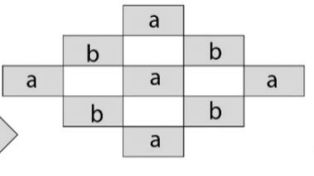

(c)

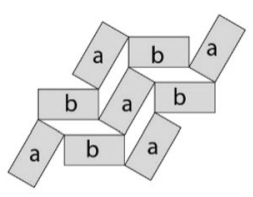

(d)

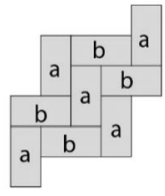

(e)

Fig. 6 The well-known herringbone pattern (wallpaper group "pg") which is moveable by use of parallel four-bar linkages

Fig. 7 This moveable system can be closed

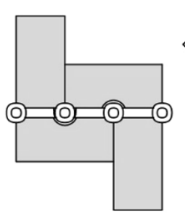

(a)

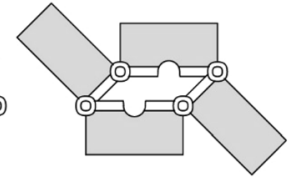

(b)

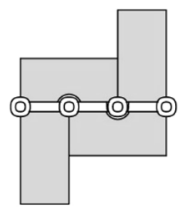

(c)
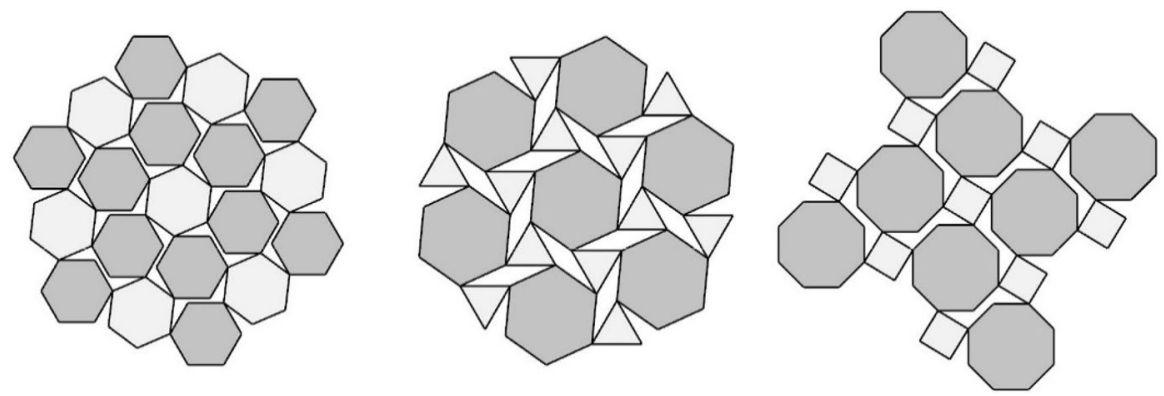

Fig. 8 One Euclidian and two Archimedean patterns with monostable auxetic behavior. Each system has a mobility with one degree of freedom

the hinges (Fig. 7). Depending on the material used and the pattern design, this scheme has to be adapted accordingly.

In order to find and create new and interesting patterns for auxetic purposes, we suggest concentrating first on basic geometric tilings of the plane and to develop them further. Figure 8 shows three of the Archimedean patterns in opened positions. These patterns allow a precise kinematic one degree of freedom motion and throughout the movement the whole system stays flat.

A much bigger variety for design purposes are given using the whole wallpaper group. But it is still an open question, which of the patterns allow a precise planar geometric motion and therefore represent monostable auxetics. Figure 9 shows examples of the wallpaper groups $\mathrm{p} 1$ and $\mathrm{p} 6$ in different opened positions. 

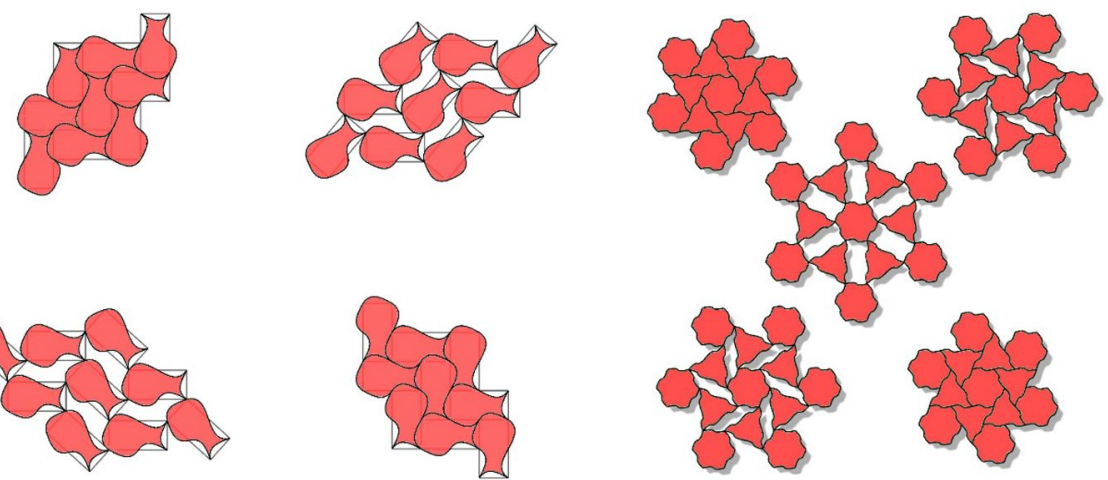

Fig. 9 Examples of the wallpaper groups p1 (left side) and p6 (right side) show auxetic behavior

\section{Bistable Auxetics}

The term bistable auxetics is used to describe systems of linked elements which are not moveable as long as rigid parts are used. In order to make the system moveable, elastic or bendable materials are used. The system always has a start position where all the parts are in one plane. If forces are applied the elements are bent and move out of the plane until they reach a stable end position, again in the plane. Due to the two stable positions, the name bistable is used.

No parallel four-bar linkages can be used as they are in the monostable cases. Instead, we could use general four-bar linkages to connect our ornamental parts. However, in this case we lose the symmetry of the motion and there is no chance to setup a closed system and connect several parts of the pattern. Additionally the motion of the parts interferes with itself. One example is shown in Figs. 10, 11 and 12. This wallpaper pattern, named p31 m, consists of triangular and arrow-shaped parts.

One solution could be to continue the motion out of plane as is shown in Fig. 11. The triangular parts rotate around horizontal axes and their edges describe cones of revolution. This configuration however, works only for parts aligned in one row. The elements of the next rows cannot be added because they do not meet the length constraint. In order to make this system work, bistable auxetics use a backdoor and take material which is bendable like paper or sheet metal. When this system is moved and

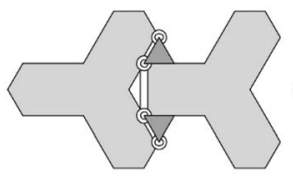

(a)

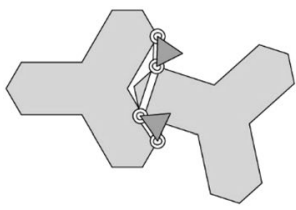

(b)

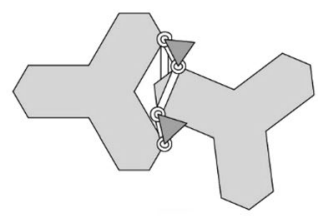

(c)

Fig. 10 A general four-bar mechanism hinders the motion of the ornamental parts and loses the symmetric configuration. No parallel four-bar linkage can be incorporated 


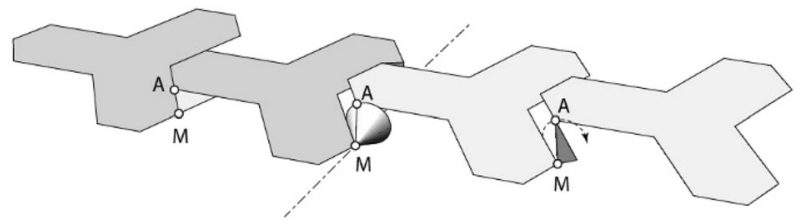

Fig. 11 For pattern parts aligned in one direction the motion can be continued into three space. The edges of the triangles describe cones of revolution with horizontal axes

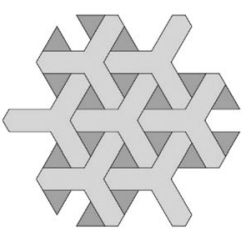

(a)

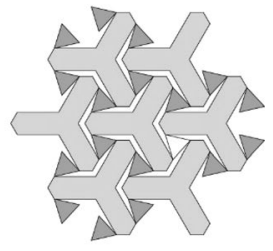

(b)

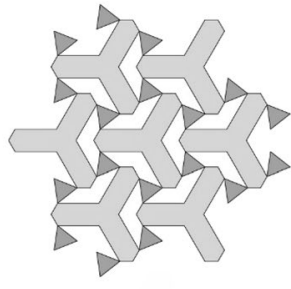

(c)

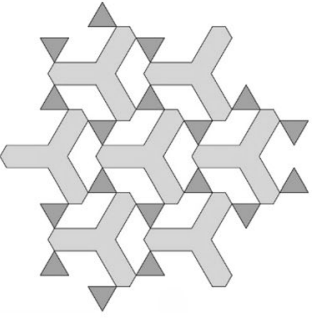

(d)

Fig. 12 The pattern p31 $\mathrm{m}$ in different opened and closed positions. But only in the very left and right position the triangles are equilateral

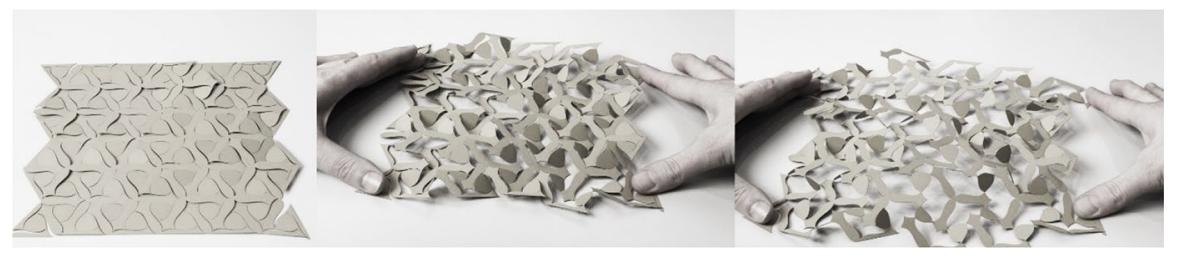

Fig. 13 The pattern p31 $\mathrm{m}$ in a more artistic design made out of paper

the parts are not rigid they are bent into the three-dimensional space and we get threedimensional forms. The drawback in this situation is that we cannot longer describe the process exactly in a kinematic way, since kinematic always describes the geometry and movement of rigid parts. The next figures demonstrates this situation. Figure 12 shows a schematic view of the pattern. On the very left and right side the system is plane and geometrically precise. In the two positions in-between, the triangles are smaller and so the pattern cannot be flat any longer. Figure 13 there is a paper version of this auxetic where we can see how the pattern parts are bent and move three-dimensionally out of the plane. 

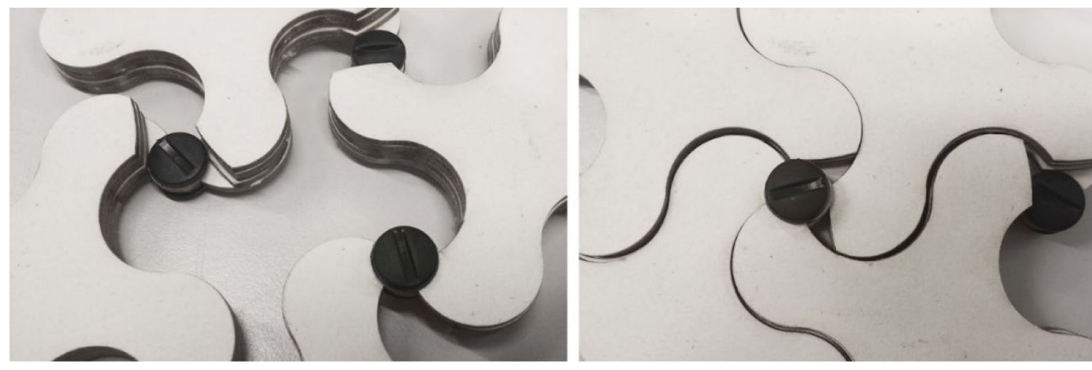

Fig. 14 A planar moveable ornamental pattern

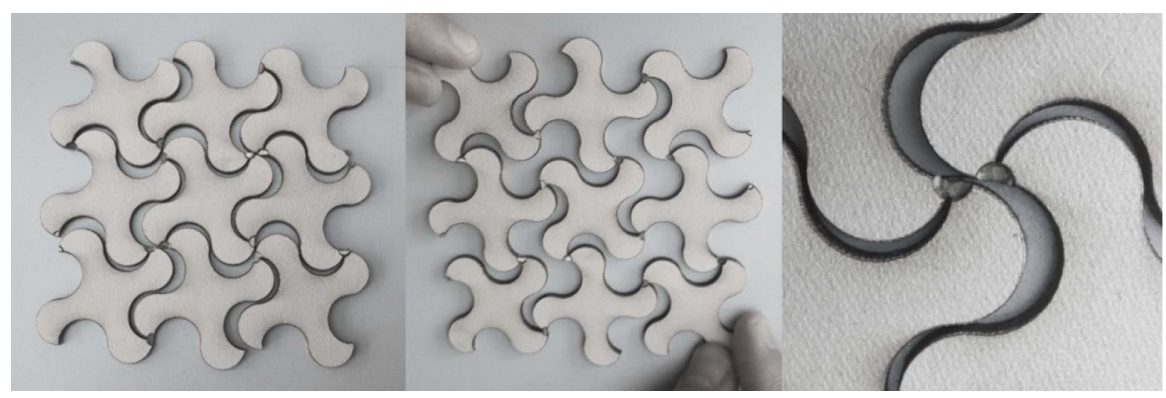

Fig. 15 One auxetic pattern built with meta-material. The different parts consist of three layers. On top and bottom of each part there is rigid cardboard which encloses flexible fabric in between. The fabric acts like a hinge (right)

\section{Joints}

In order to fabricate an auxetic structure, revolute and spherical joints can be used, especially for mechanical purposes. Figure 14 shows a version with revolute joints. One challenge of this is always to find a feasible solution, so that the joints enable a motion to the closed positions. A alternative choice, especially for designer's purposes, is the use of so called "auxetic meta-material" which is a composite of two different materials. A rigid one keeps the form of the pattern parts and a flexible one allows the rotations and acts like a hinge (Fig. 15). The parts consists of three layers. On top and bottom the rigid material is used and in between the flexible one is incorporated and connects the different parts with each other.

\section{From Kinematic Structure to Parametric Design}

In the first step, for the design of a variety of auxetic structures we choose a "simple" kinematic system which is moveable with at least one degree of freedom. Simple means that the system usually consists of one or two types of congruent 
polygons, not necessarily regular. The polygons are connected by congruent parallel (diamond-shaped) four-bar linkages. Figures 2 and 5 show quadrangles and diamond-shaped four-bar linkages. Figure 6 shows rectangles and parallel four-bar linkages. The patterns in Fig. 8 include triangles, quadrangles, hexagons and octagons.

In a second step, the polygon-outlines are replaced by curves; in most cases these are NURBS curves (Farin 2001). This can be done arbitrarily in a certain range, whereby it must be ensured that the individual elements fit together and the mechanism can be opened and closed. This can be done by means of the theory of the wallpaper groups. In order to vary the curved outlines, the control points of the NURBS curves should be defined parametrically. This leads to examples like those in Figs. 3 and 9. So the underlying kinematic system of such an auxetic structure always stays the same, only the outline shape of the individual forms change.

As long as the underlying kinematic system is moveable and planar, we get moveable and planar auxetic forms. Conversely, the geometric pattern in the Figs. 10, 11, 12 and 13, is not moveable in a kinematic sense, since kinematic always deals with rigid bodies. The way out for this situation is use flexible materials and to bend the individual parts.

\section{Design Examples}

Figure 16 shows an auxetic pattern which is based on the kinematic system shown in Fig. 5. This means that linked and congruent diamond-shaped four-bar linkages define the movement. The geometric basic form - that is a quadrangle-is replaced by arbitrary polygons. The special thing about this form is that every quadrangle is replaced by another polygon. There is a kind of evolution visible in this, from the original quadrangle to the arbitrary polygon shape, from the left to the right and from the bottom to the top (Fig. 16). Such patterns are also called a-periodical auxetic structures.

In order to make the system moveable we did not cut the whole border of each element but left a small amount of material in place of the connections. Since these connections are very small they can act like revolute joins (Fig. 16, right side).

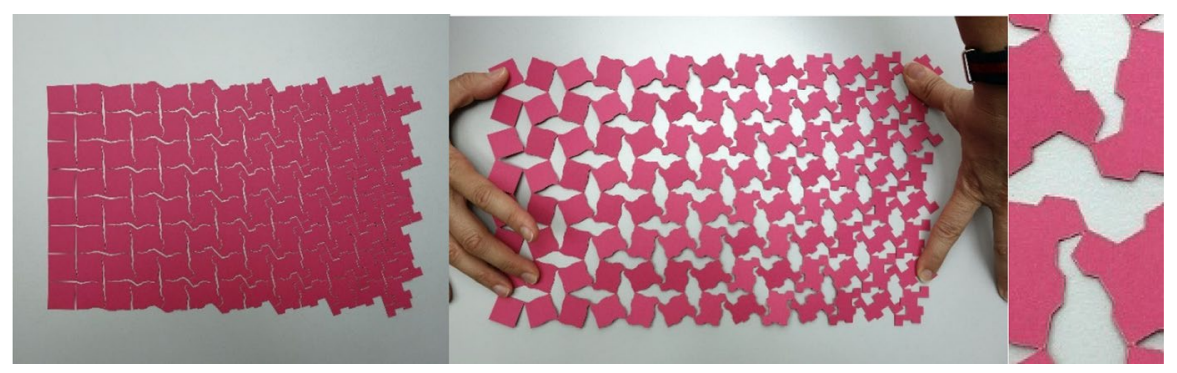

Fig. 16 A monostable a-periodical auxetic structure based on the wallpaper pattern p4 like in Fig. 2 


\section{Conclusion}

Auxetic materials and auxetic behavior is very effective not just for practical use but also for design purposes in architecture, such as façade elements, screens or shading systems. Since our approach to the matter is a geometric one we have explained the structure of such materials in a precise way, without computer simulation or approximation. This works most of all for monostable auxetics, whose behavior can be descripted in a precisely geometric way. As a base we used Platonic and Archimedean patterns as well as the wallpaper groups. The examples shown in this paper, which can be described precisely in a geometric way, can be scaled to any size. This is the great advantage compared to auxetic structures used in material sciences, which use elastic material properties which they explore in a more experimental way. For application purposes in a bigger scale, the joint system has to be considered and solved, since adjacent parts often interfere. Finally, there is still an open question as to which of the wallpaper groups can be used to construct an efficient auxetic structure, either monostable or bistable. Also three-dimensional auxetic structures, which are not discussed in this paper, could be a valuable subject for further research. There already exist examples of such structures and a deeper analysis would be worthwhile. Future work will be to investigate applications for architectural design, especially in shading systems and façade elements.

Acknowledgements Open access funding provided by Graz University of Technology.

OpenAccess This article is distributed under the terms of the Creative Commons Attribution 4.0 International License (http://creativecommons.org/licenses/by/4.0/), which permits unrestricted use, distribution, and reproduction in any medium, provided you give appropriate credit to the original author(s) and the source, provide a link to the Creative Commons license, and indicate if changes were made.

\section{References}

Borcea, Ciprian. and Ileana Streinu. 2015. Geometric auxetics, Proceedings of The Royal Society A Mathematical Physical and Engineering Sciences 471(2184).

Bottema, Oene. and Bernard Roth 1979. Theoretical Kinematics. North-Holland Series in Applied Mathematics and Mechanics 24. Amsterdam-New York-Oxford.

Elipe, Juan Carlos Álvarez and Andres Diaz Lantada. 2012. Comparative study of auxetic geometries by means of computer-aided design and engineering, Smart Materials and Structures, 21(10), https://doi.org/10.1088/0964-1726/21/10/105004.

Ellul, Brian. Martin Muscat and Joseph Grima. 2009. On the effect of the Poisson's ratio (positive and negative) on the stability of pressure vessel heads, Physica Status Solidi B: Basic Research, 246(9): 2025-2032.

Evans, Ken. and Andrew Alderson. 2000. Auxetic materials: Functional materials and structures from lateral thinking. Advanced Materials, 12(9): 617-628.

Farin, Gerald. 2001. Curves and Surfaces for CAGD: A Practical Guide. San Francisco: Morgan Kaufmann Publisher. 
Gatt, Ruben. Luke Mizzi, Joseph Azzopardi, Keith Azzopardi, Daphne Attard, Aaron Casha, Joseph Briffa and Joseph Grima. 2015. Hierarchical Auxetic Mechanical Metamaterials. Sci. Rep. 5, 246(9):2025-2032. https://doi.org/10.1002/pssb.200982033.

Hawkes, Elliot, Byoungkwon An, Nadia Benbernou, H.Tanaka, Sangbae Kim, Erik Demaine, Daniela Rus and R. J Wood. 2010. Programmable matter by folding. Proceedings of the National Academy of Sciences 107(28):12441-12445.

Konaković, Mina. Keenan Crane, Bailin Deng, Sofien Bouaziz, Daniel Piker and Mark Pauly. 2016. Beyond Developable: Computational Design and Fabrication with Auxetic Materials, ACM Transactions on Graphics, 35(4):1-11.

Lakes, Roderic. 1987. Foam structures with a negative Poisson's ratio, Science, 235: 1038-1040.

Mesa, Olga. Milena Stavrić, Saurabh Mhatre, Jonathan Grinham, Sarah Norman, Allwn Sayegh and Martin Bechthold. 2017. Non-Linear Matters: Auxetic Surfaces. In: Proceedings of ACADIA, 392-403, MIT, Cambridge, USA.

Najabat, Murtaza. James Busfield and Ihtesham Rehman. 2014. Auxetic oesophageal stents: structure and mechanical properties, J. Mater. Sci: Mater Med., 25: 527-553.

Pottmann, Helmut. Andreas Asperl, Michael Hofer and Axel Kilian. 2007. Architectural Geometry. Exton PA: Bentley Institute Press.

Rafsanjani, Ahmad. and Damiano Pasini. 2016. Bistable auxetic mechanical metamaterials inspired by ancient geometric motifs, Extreme Mechanics Letters, 9: 291-296.

Sanami, Mo. Naveen Ravirala, Kim Alderson and Andrew Alderson. 2014. Auxetic materials for sports applications. Procedia Engineering, 72: 453-458.

Tan, Tho Wei. Graeham Douglas, Timothy Bond and Srikantha Phani. 2011. Compliance and longitudinal strain of cardiovascular stents: influence of cell geometry. Journal of Medical Devices 5(4):041002 (https://doi.org/10.1115/1.4005226).

Publisher's Note Springer Nature remains neutral with regard to jurisdictional claims in published maps and institutional affiliations.

Milena Stavric $\mathrm{PhD}$, is a teacher and researcher at Graz University of Technology. She obtained a $\mathrm{PhD}$ (Architecture) with the focus on higher order geometrical curves in architecture from University of Belgrade. She is interested in geometric structures and their application in architecture.

Albert Wiltsche $\mathrm{PhD}$, is a teacher and researcher at Graz University of Technology. He obtained a PhD in Geometry with the focus on freeform structures. He has a strong interest in geometric architecture. 